\section{Spring greens}

This edition of marketplace includes vectors encoding autofluorescent proteins, a microscopic fluorescence module, a PCR-sequencing robot and practical courses on a wide range of techniques.

With spring in the air and the proverbial green shoots soon to appear, one's thoughts inevitably turn to the bi-annual product review, which springs forward with six new vectors encoding autofluorescent proteins (AFPs $^{\mathrm{TM}}$ ) supplied by Quantum Biotechnologies Inc. These incorporate cDNA encoding either the green or blue fluorescent proteins, allowing for easy detection. For transfection of mammalian cells, the pQBI25 and pQBI50 vectors encode green and blue fluorescent proteins, respectively, and are under the control of the CMV promoter and can be used in transient expression, trafficking and localization studies or as reporter vectors (upon substitution of the promoter). As positive selection markers, $\mathrm{pQBIPGK}$ and $\mathrm{pQBIPoll}$ vectors encode the green fluorescent protein (GFP) with neomycin resistance under the control of the murine PGK and PolII promoters, respectively ... and for overexpression of proteins in E. coli, pQBI63 encodes GFP, and PQBI67 encodes a blue fluorescent protein, both under the control of the $T 7$ promoter.

To visualize fluorescently-labelled specimens, Leica Inc. has marketed a fluorescence module, which, in conjunction with their stereomicroscope LEICA MZ12, enables unprepared fluorescing specimens to be inspected, manipulated, sorted and recorded. The intense light produced by its mercury vapour lamp, together with specific filter sets, enables fine structure such as individual nerve cells - to be differentiated. The module can be fitted onto any of the $\mathrm{M}$-series microscopes and to some other models as well.

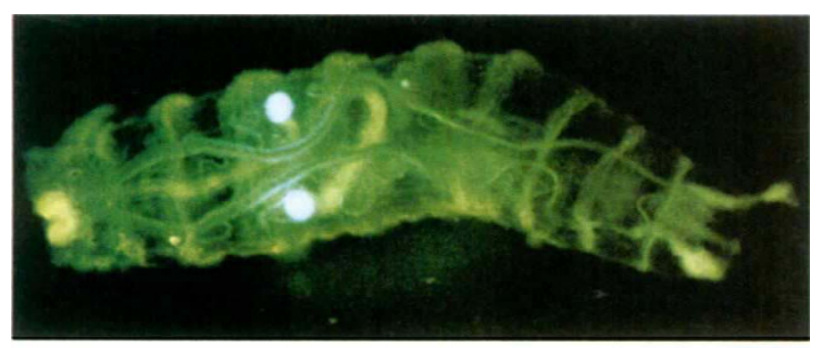

Using the fluorescence module marketed by Leica Inc., S. Wang and T. Hazelrigg obtained this image of a third instar male Drosophila larva.

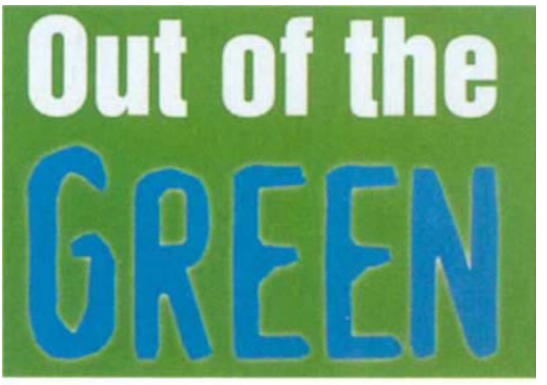

Quantum Biotechnologies Inc. market vectors encoding autofluorescent proteins.

A new DNA isolation kit designed to recover high-molecular weight genomic DNA $(100-500 \mathrm{~kb})$ has been marketed by

Stratagene. DNA can be isolated from whole tissues such as liver, spleen, kidney and brain, replacing standard phenol/chloroform extractions. One hour of handson time followed by an overnight incubation and voila! Fully hydrated DNA at one's disposal. Should it require amplification, the new Expand ${ }^{\mathrm{TM}} 20 \mathrm{~kb}$ PLUS PCR system by Boehringer Manneheim will PCR-amplify lambda DNA fragments up to $35 \mathrm{~kb}$ with good fidelity and yield, achieved by combining $P$ wo and Taq DNA polymerases with a novel enzymatic component and an 'activated' reaction buffer.

For those with a heavy demand for PCR and sequencing capacity, Perkin Elmer has produced the ABI PRISM 877 Integrated Thermal Cycler - an automated workstation referred to as a 'robot'. The work surface integrates a 384-well thermal cycler with a high-precision robot capable of pipetting extremely low volumes necessary for both PCR and sequencing reactions, which can be carried out on a single platform. Complete automation enables high throughput for a variety of applications. The system can run thousands of PCR and DNA sequencing samples a day and can also run overnight, allowing one to spend time on more productive tasks such as skiving off and enjoying the beauties of spring-time.

Tired of blue fingers and that moment of suspense when trying to siphon off gel stain without pouring the gel down the drain? Then Pharmacia's Hoefer Automated Gel Stainer will come as welcome news; this allows for completely automated and reproducible staining of gele and is suitable for Coomassie blue and silver staining. A wide variety of gels, including plastic-baclied and standard vertical polyacrylamide gels of varying size, can be accommodited. After connecting the staining whutions to the system, the gel is placed in the staining tray and one of eight pre-programmed or twenty personalized staining protocols is selected. With the push of a button, the procedure is completed.

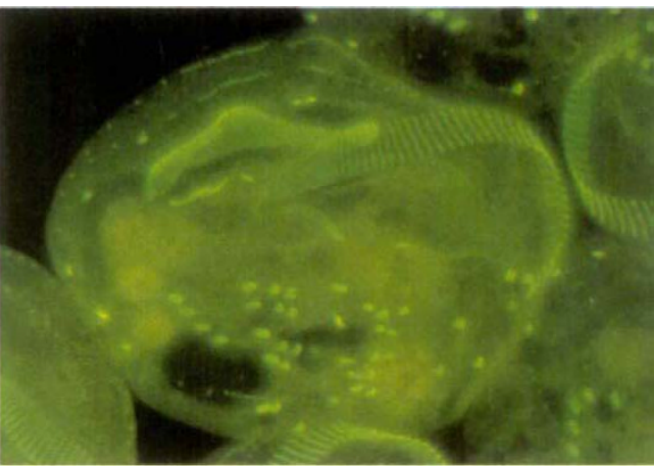

Go on an ATCC course and learn how to obtain images like ohn Koetzel of the University of Maryland. The cells above e of the ciliate Euplotes.

Finally, if you're feeling blue because you're feeling green, turn down that Green Day tape and sign up for one of the American Type Culture Collection (ATCC) educational training workshops. Brush up on recombinant DNA techniques, $P C R$ applications, DNA fingerprinting, in situ hybridization, microscopy, cell and hybridoma culture, antibody production, patenting and more. These are 'hands-on' workshops, with about half the time spent on practical work and the other half on lectures. They are geared towards research and industrial scientists, technicians, biotechnologists and patent attorneys. You'll be greening from ear to ear!

Notes compiled by AETTE PMIMISTER

For more information, call:

Quantum Biotechnologies 1-888-362-5487 • Leica 1-847-405-0123 - Stratagene 1-800-424-5444 • Boehringer Mannheim 1-800-428-5433 • Perkin-Elmer 1-203-762-1000 • Pharmacia +46 18 165000 ATCC 1-301-881-2600 